\title{
Microstructure dependence of diffusional transport
}

\author{
Jingzhi Zhu ${ }^{\mathrm{a}, *}$, Long-Qing Chen ${ }^{\mathrm{a}}$, Jie Shen ${ }^{\mathrm{b}}$, Veena Tikare ${ }^{\mathrm{c}}$ \\ a Department of Materials Science and Engineering, 231 Steidle Building, Penn State University, University Park, PA 16802, USA \\ b Department of Mathematics, Penn State University, University Park, PA 16802, USA \\ ${ }^{\mathrm{c}}$ Materials Modeling and Simulation, Sandia National Laboratory, Albuquerque, NM 87185-1411, USA
}

Received 2 February 2000; received in revised form 10 May 2000; accepted 30 May 2000

\begin{abstract}
A simple and effective numerical method is proposed for simulating the temporal diffusive mass transport process through a microstructure with arbitrary complexity described by a phase-field approach. The mass diffusion through a given microstructure is modeled by a diffusion equation with a variable diffusion coefficient, which is solved by an efficient and accurate semi-implicit spectral method. It is shown that it is possible to extract the effective diffusion coefficient for any given microstructure from the temporal concentration profiles. The method is used to simulate the grain boundary diffusion in a single-phase polycrystalline grain structure and the heterogeneous diffusion in a twophase microstructure with different diffusion coefficient in each phase. Results are compared with existing analytical theories and computer simulations. (c) 2001 Elsevier Science B.V. All rights reserved.
\end{abstract}

PACS: 66.30.-h; 02.60.Cb; 66.10.Cb

Keywords: Diffusion; Microstructures; Phase-field; Spectral method; Grain boundary diffusion; Effective diffusion coefficient

\section{Introduction}

Diffusion in a solid with a multi-phase microstructure or with defects such as grain boundaries and dislocations can be highly heterogeneous. For example, in a polycrystalline grain structure, the diffusion coefficients along a grain boundary and through a bulk lattice can be orders of magnitude different. As a result, although the volume fraction of grain boundaries is small, grain boundary diffusion may dominate a number of processes such as grain growth, micro-electronics device failure,

\footnotetext{
${ }^{*}$ Corresponding author. Tel.: +1-814-863-9957; fax: +1-814867-0476.

E-mail address: jzhu@psu.edu (J. Zhu).
}

and sintering [1]. Macroscopically, the effective diffusion coefficient of a grain structure depends on the relative magnitudes of the grain boundary and bulk diffusion coefficients, the average grain size and grain boundary width. Although it is possible to derive analytical solutions for very special microstructures, it is impossible to derive the effective properties of a microstructure exactly. Therefore, most of the statistical theories are concerned with the property bounds [2]. Recently, there has been increasing interest in computing the effective properties from a microstructure either from a digitization of an experimental microstructure or from a microstructure simulation model [3]. However, most of these approaches are based on steady-state equations and thus cannot describe the temporal mass diffusive transport behavior. 
The purpose of this paper is to develop a simple and effective numerical method to simulate the temporal diffusive transport through microstructures with arbitrary complexity. A microstructure is described by field variables and generated by the phase-field method. A semi-implicit FourierChebyshev spectral method was proposed to efficiently solve the diffusion equation with a variable diffusion coefficient. It will be shown that, using this method, both the temporal diffusion profile through a microstructure and the macroscopic effective diffusivity can be obtained. It is used to simulate the diffusion through a single-phase grain structure and two-phase microstructures with different diffusive properties in each phase.

\section{Computer modeling}

\subsection{Microstructure description}

To model the diffusion through an arbitrary microstructure, we used a set of space- and timedependent field variables to describe the microstructure, similar to the phase-field approach [4]. For example, the concentration difference throughout the microstructure is described by a concentration field $C(\mathbf{r})$. A single-phase polycrystalline microstructure can be described by a set of order-parameter field variables, $\eta_{1}(\mathbf{r}), \eta_{2}(\mathbf{r}), \ldots$, $\eta_{p}(\mathbf{r})$ where $p$ is the number of grain orientations in the system [5].

To simplify the problem, we studied diffusion in a static microstructure which remained unchanged during the diffusion process. The solid state reactions between the diffusant atoms and the microstructure such as segregation, which may occur in some practical applications, are not considered in this work. Therefore, only temporal and spatial evolution of one variable, the concentration of diffusing species, is needed to investigate the diffusive transport in a microstructure described by a set of static field variables. The spatial dependence of diffusivity is introduced through its dependence on the field variables $C(\mathbf{r})$ or $\eta(\mathbf{r})$, i.e., we describe the diffusion coefficient $D$ as a function of $C$ or $\eta$ written as $D=f\left(C, \eta_{1}, \eta_{2}, \ldots, \eta_{p}\right)$. For example, in a single-phase polycrystalline material, the diffu- sion coefficient throughout the system can be expressed in a scaled form as $D=D^{*}\left(1-a \sum_{i=1}^{p} \eta_{i}^{2}\right)$, where $D^{*}$ and $a$ are positive constants. The values of $\sum \eta_{i}^{2}$ in the grain bulk are higher than those in the grain boundaries, and therefore the difference in grain boundary and bulk diffusion coefficients can be easily described by this simple equation. Although the choice of this equation was quite arbitrary, we believed it would not affect the general results of the diffusive transport process. One important advantage is the fact that it avoids the specification of boundary conditions at the interfaces between different regions with different diffusivites. Since there was no microstructural evolution in our study, the variable $\sum \eta_{i}^{2}$ was only a function of spatial coordinates $\mathbf{r}$, and thus the diffusion coefficient was mapped to each lattice point as $D(\mathbf{r})$. It should also be pointed out that the field variables are not required to be diffuse as they are in the phase-field model.

\subsection{Numerical method}

Neglecting the segregation of diffusing species, the temporal diffusive transport process through the microstructure can be described by Fick's second law written as

$\frac{\partial C(\mathbf{r}, t)}{\partial t}=\nabla \cdot D(\mathbf{r}) \nabla C(\mathbf{r}, t)$,

where $C$ is the concentration of diffusant, $t$ the time, $\mathbf{r}$ the spatial coordinate, and $D(\mathbf{r})$ is the diffusion coefficient distribution in a given microstructure. Our numerical simulation was performed in two dimensions with a periodic boundary condition in one-dimension and a fixed boundary condition in the other which has a fixed diffusant source and sink. The initial concentration of diffusant throughout the system is zero.

Analytical solutions to the above equation are only possible with highly idealized assumptions about the diffusion coefficient distribution $D(\mathbf{r})$. For studying a distribution with arbitrary complexity, numerical simulations have to be employed. Most of the existing numerical simulations for this problem were performed using explicit forward Euler method in time and finite-difference in space. To maintain the stability of the scheme 
and to achieve high accuracy, the time step and spatial grid size have to be very small, which seriously limit the system size and time duration of a simulation. Spectral methods, which were widely used in the field of fluid dynamics [6], offered us an accurate and efficient alternative to solve partial differential equations. Recently, Chen and Shen [7] used a semi-implicit Fourier spectral method for solving the time-dependent Ginzburg-Landau and Cahn-Hilliard equations. They showed that the semi-implicit Fourier spectral method is significantly more efficient and accurate than a conventional explicit finite difference method.

Consider the forward Euler method for Eq. (1)

$\frac{C^{n+1}-C^{n}}{\Delta t}=\nabla \cdot D \nabla C^{n}$.

The above scheme with a finite difference or Fourier approximation in space has a severe time step constraint of the form $\Delta t \leqslant \lambda / N^{2}$ [6], where $\lambda$ is a constant and $N$ is the number of lattice points to be used in each space dimension. The constraint becomes $\Delta t \leqslant \lambda / N^{4}[6]$ when the problem has a fixed boundary and a spectral approximation is used in space. To avoid such a severe restriction, we consider a semi-implicit treatment. The idea is to split the variable diffusion coefficient $D(\mathbf{r})$ into $A$ and $D(\mathbf{r})-A$, where $A$ is a suitable constant, and treat them separately to obtain

$\frac{C^{n+1}-C^{n}}{\Delta t}-A \nabla^{2} C^{n+1}=-\nabla \cdot(A-D) \nabla C^{n}$.

write $u=C^{n+1}, \alpha=1 /(A \Delta t)$, and $f=C^{n} /(A \Delta t)-$ $\nabla \cdot(1-(D / A)) \nabla C^{n}$, and then Eq. (3) can be simplified as

$\alpha u-\nabla^{2} u=f$,

with a periodic boundary condition in one direction and a fixed boundary condition in the other. At each time step, this equation can be solved accurately and efficiently by a Fourier-Chebyshev Galerkin algorithm which we briefly describe below.

Expanding $u$ and $f$ in Fourier series in the $x$ direction in which a periodic boundary condition is applied, and substituting $u(x, y)=\sum_{m=-\infty}^{\infty} u^{m}(y)$ $e^{i m x}$ (same for $f$ ) in Eq. (4), we obtain

$$
\begin{aligned}
& \left(\alpha+m^{2}\right) u^{m}-u_{y y}^{m}=f^{m} \\
& m=0, \pm 1, \pm 2, \ldots ; \\
& u^{m}( \pm 1)=0
\end{aligned}
$$

Then, we solve the above equation for each $m$ by using the Chebyshev-Galerkin algorithm developed by Shen [8]. More precisely, let $T_{k}(y)$ be the $k$ th degree Chebyshev polynomial, we look for an approximation of $u^{m}$ in the form $u_{N}^{m}=$ $\sum_{k=0}^{N-2} \hat{u}_{k}^{m} \phi_{k}(y)$ where $\phi_{k}(y)=T_{k}(y)-T_{k+2}(y)$ are the Galerkin basis functions satisfying $\phi_{k}( \pm 1)=0$. Let us denote $(u, v)_{\omega}=\int_{-1}^{1} u v \omega \mathrm{d} y$ where $\omega=$ $\left(1-y^{2}\right)^{-(1 / 2)}$ is the Chebyshev weight function. Then, the coefficients $\left\{\hat{u}_{k}^{m}\right\}_{k=0}^{N-2}$ (hence the approximation $u_{N}^{m}$ ) are determined by the weighted variational formulation for Eq. (5)

$$
\begin{aligned}
& \left(\alpha+m^{2}\right)\left(u_{N}^{m}, \phi_{k}\right)_{\omega}-\left(\left(u_{N}^{m}\right)_{y y}, \phi_{k}\right)_{\omega} \\
& \quad=\left(I_{N} f^{m}, \phi_{k}\right)_{\omega}, \quad k=0,1,2, \ldots, N-2,
\end{aligned}
$$

where $I_{N}$ is a polynomial interpolation operator based on the Chebyshev-Gauss-Lobatto (CGL) points: $y_{j}=\cos (j \pi / N), j=0,1, \ldots, N$. More precisely, for any continuous function $g$ on $[-1,1]$, $I_{N} g$ is the unique polynomial of degree less or equal than $N$ such that

$I_{N} g\left(y_{j}\right)=g\left(y_{j}\right), \quad j=0,1, \ldots, N$.

Furthermore, the transform between the function values $\left\{f\left(y_{j}\right)\right\}_{j=0}^{N}$ and the Chebyshev coefficients of $I_{N} f$ can be evaluated in $\mathrm{O}(N \log N)$ operations by using the fast Fourier transform (FFT).

Now, we describe how to determine the coefficients $\left\{\hat{u}_{k}^{m}\right\}_{k=0}^{N-2}$ from Eq. (6). Let us denote

$$
\begin{aligned}
& f_{k}=\int_{-1}^{1} I_{N} f \phi_{k} \omega \mathrm{d} y, \quad \bar{f}=\left(f_{0}, f_{1}, \ldots, f_{N-2}\right)^{\mathrm{T}}, \\
& s_{k j}=-\int_{-1}^{1} \phi_{j}^{\prime \prime} \phi_{k} \omega \mathrm{d} y, \quad S=\left(s_{k j}\right)_{k, j=0,1, \ldots, N-2}, \\
& b_{k j}=\int_{-1}^{1} \phi_{j} \phi_{k} \omega \mathrm{d} y, \quad B=\left(b_{k j}\right)_{k, j=0,1, \ldots, N-2} .
\end{aligned}
$$

Then, it is easy to see that Eq. (6) is equivalent to the following linear system

$\left[\left(\alpha+m^{2}\right) B+S\right] \bar{u}=\bar{f}$, 
where $\bar{u}=\left(\hat{u}_{0}^{m}, \hat{u}_{1}^{m}, \ldots, \hat{u}_{N-2}^{m}\right)^{\mathrm{T}}$. It is shown in [8] that the entries of the matrices $S$ and $B$ are given by:

$s_{k k}=2 \pi(k+1)(k+2), \quad k=0,1, \ldots, N-2$,

$s_{k j}=4 \pi(k+1), \quad j=k+2, k+4, k+6, \ldots$,

$s_{k j}=0, j<k$ or $j+k$ odd,

and

$b_{k k}=\pi, \quad k \neq 0, \quad$ and $\quad b_{00}=2 \pi$,

$b_{k j}=-\frac{\pi}{2}, \quad j=k \pm 2$,

$b_{k j}=0, \quad$ otherwise.

Although $S$ is not sparse but it is a special upper-triangular matrix whose non-zero elements (except the diagonal element) at each row are all the same. Therefore, one can design a special Gaussian elimination process which takes into account this special structure to solve Eq. (7) in $\mathrm{O}(N)$ operations (more precisely, at a cost comparable to that of solving a penda-diagonal matrix). We refer to [8] for more details on this matter.

Thus, the overall operation counts for solving Eq. (5) is $\mathrm{O}(N \log N)$ for each $m$. Hence, assuming $M$ Fourier coefficients are retained in the Fourier expansion, the total cost of the FourierChebyshev Galerkin method for solving Eq. (4) is $\mathrm{O}(N M \log (N M))$ which is quasi optimal with respect to the number of points (lattices) used. Note that the total cost is only slightly more than $\mathrm{O}(N M)$ operations which are required by the conventional finite difference/finite element methods, but the Fourier-Chebyshev Galerkin method is capable of producing much more accurate results using significantly less number of points (lattices). On the other hand, the semiimplicit treatment will allow us to use much larger time steps than that is allowed by an explicit method (about two orders of magnitude larger from our numerical experiments). Hence, the resulting semi-implicit Fourier-Chebyshev Galerkin method is an extremely efficient method for solving diffusion equation with a variable diffusion coefficient.

\section{Results and discussion}

\subsection{Grain boundary diffusion}

By numerically solving the diffusion equation with a variable coefficient, we can obtain the temporal evolution of the concentration profile through any arbitrary microstructure. The first example is the grain boundary diffusion, which has been studied for many years since the early 50s. For an idealized grain boundary geometry, analytical solutions are available for the concentration profiles [9-11]. These solutions can be employed to analyze the grain boundary diffusion coefficients from experimental measurements [12]. In recent years, some numerical models were developed to overcome the simple geometry and topology restrictions. Gui et al. [13] analyzed the grain boundary diffusion process in thin films by transmission-line-matrix modeling . More recently, Swiler et al. [14,15] investigated some important heterogeneous diffusion effects in polycrystalline microstructures obtained from Potts model simulations.

We first tested our approach by studying the diffusion through an ideal grain boundary described by the Fisher's model where the analytical results were available [9]. The grain boundary is treated as a high-diffusivity, semi-infinite isotropic slab of uniform width, embedded in a low-diffusivity, semi-infinite perfect crystal which is normal to the surface that carries the diffusant shown in Fig. 1(a). However, in numerical simulations we can approximate a grain boundary as a finite slab embedded in a finite crystal. This approximation can represent a semi-infinite system very well if the grain boundary thickness $\delta$ is far less than the system size $L(\delta \ll L)$. A simple way to describe such a grain boundary in Fisher's model is to choose two field variables $\eta_{1}, \eta_{2}$. In grain $1, \eta_{1}=1$, $\eta_{2}=0$. In grain $2 \eta_{1}=0, \eta_{2}=1$. The phase field model applied to describe the microstructure usually involves a diffusive interface, where the field variables $\eta_{1}, \eta_{2}$ change continuously from 0 to 1 across the grain boundary, shown in Fig. 1(b). The solid line in the Fig. 1(b) plotted the diffusion coefficient $D$ across the grain boundary described by $D=D^{*}\left[1-a\left(\eta_{1}^{2}+\eta_{2}^{2}\right)\right]$ where $D^{*}=1$ 


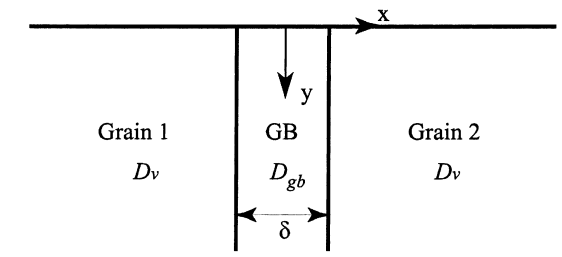

(a)
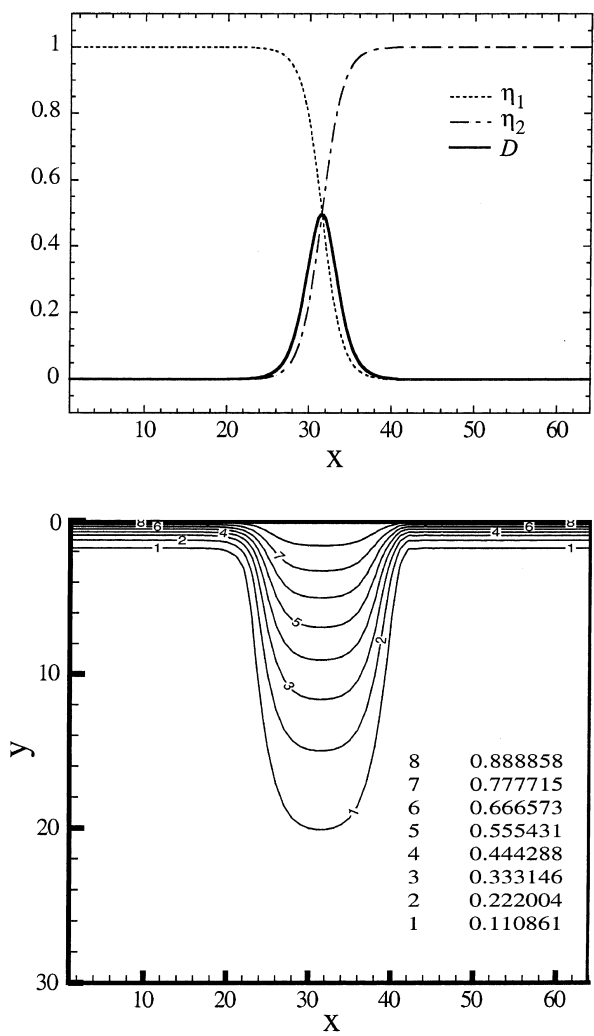

(b)

Fig. 1. Diffusion through an idealized grain boundary: (a) Fisher's model for grain boundary diffusion; (b) field variables $\eta_{1}, \eta_{2}$ and diffusion coefficient $D$ across a grain boundary in a phase-field description; (c) an isoconcentration contour plot with eight levels through a grain boundary after a certain diffusion time, $D=D^{*}\left[1-a\left(\eta_{1}^{2}+\eta_{2}^{2}\right)\right], D^{*}=1$ and $a=0.999$.

and $a=0.999$. Fig. 1(c) shows an isoconcentration contour plot through a grain boundary after a certain diffusion time. The diffusion coefficient within the grain boundary being higher than in the crystal, the diffusion penetrated deeper along the grain boundary than in the grain bulk. Consequently, it started leaking through the two walls of the grain boundary into the grain bulk.
The kinetics of grain boundary diffusion are usually classified to type A, B, and C according to the parameters of the model, such as the ratio of grain boundary diffusion coefficient to the bulk lattice diffusion coefficient, the diffusion time, and the ratio of grain boundary width to the grain size [16]. We have carried out simulations in all three regimes and compared the concentration profile at different times obtained from our simulations with the analytical solutions in each regime. The A regime is for long diffusion time $t$ and small grain size $d$ with the condition: $\left(D_{v} t\right)^{1 / 2} \gg d$. The concentration profiles in type A kinetics appear to obey Fick's law for a homogeneous system with an effective coefficient $D_{\text {eff }}$ which can be written as

$\langle C\rangle=\operatorname{erfc}\left(\frac{y}{2 \sqrt{D_{\text {eff }} t}}\right)$,

where $D_{\text {eff }}=f D_{\mathrm{gb}}+(1-f) D_{v}$ and $f$ is the volume fraction of grain boundaries. The comparison of the average concentration $\langle C\rangle$ at different times in A regime was shown in Fig. 2(a) where our numerical results (shown in symbols) agree very well with analytical solutions from Eq. (8) (shown in lines).

Type $\mathrm{C}$ kinetics is the opposite of type A kinetics where volume diffusion in the grain bulk is negligible, i.e., $\left(D_{v} t\right)^{1 / 2} \ll \delta$. Therefore the diffusion is only one-dimensional in Fig. 1(a) (along $y$ direction). With the defined boundary condition, the concentration along the grain boundary in Fig. 1(a) is

$C(y, t)=\operatorname{erfc}\left(\frac{y}{2 \sqrt{D_{\mathrm{gb}} t}}\right)$.

Fig. 2(b) showed a good agreement of the numerically calculated and analytical results of the concentration along the grain boundary in regime C.

Type B kinetics is of the intermediate case between $\mathrm{B}$ regime and $\mathrm{C}$ regime with the condition $\delta \ll\left(D_{v} t\right)^{1 / 2}<d / 2$. Most experimental conditions fall into this regime. The analytical solution in the $\mathrm{B}$ regime was obtained by Whipple in a complex form [10]. The distribution of concentration through the microstructure is described by 

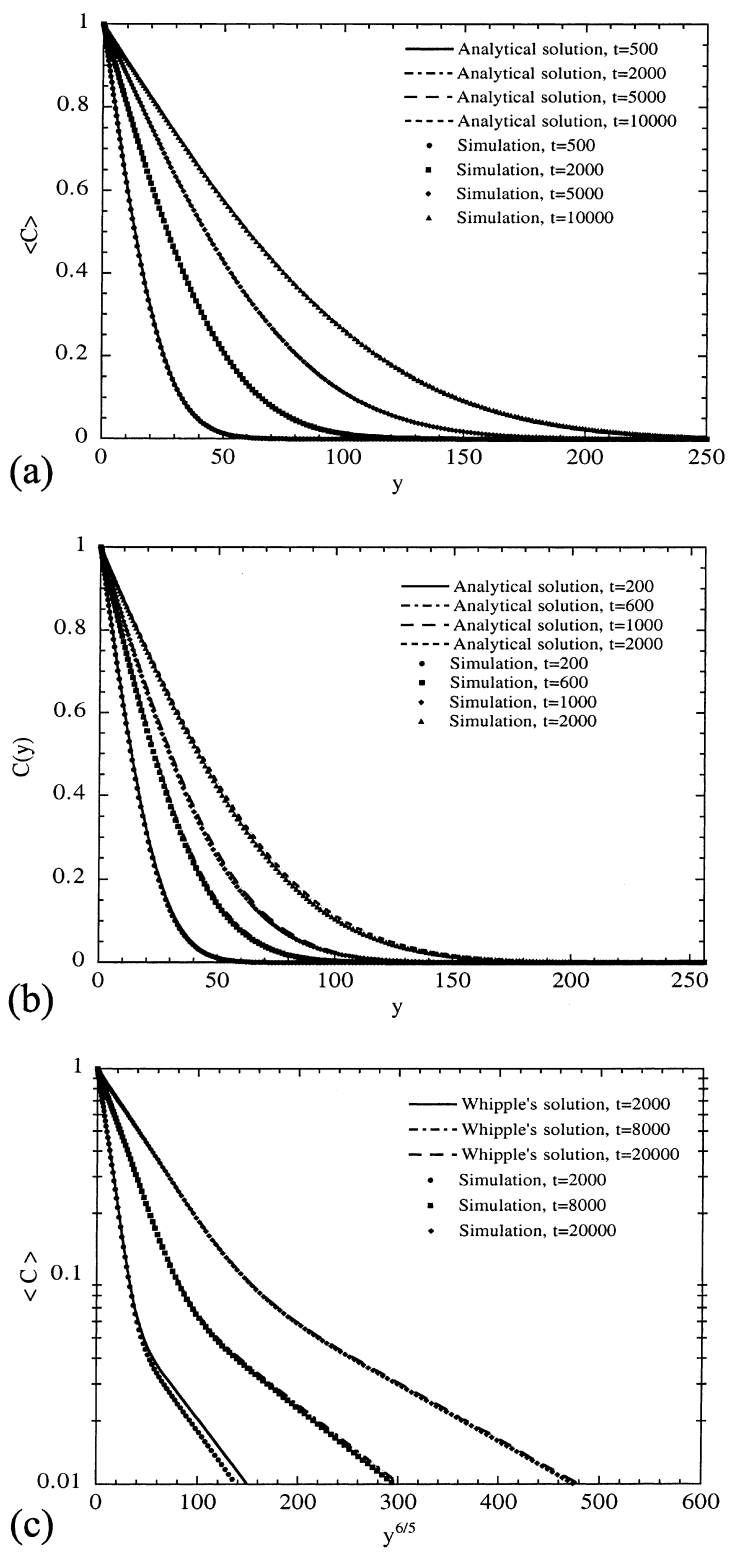

Fig. 2. Comparison of the concentration profile at different diffusion distance $y$ for a grain boundary diffusion at different diffusion times: (a) type A kinetics, $\langle C\rangle$ vs $y$; (b) type $\mathrm{C}$ kinetics, $C(y, t)$ vs $y$; (c) type B kinetics, $\langle C\rangle$ vs $y^{6 / 5}$.

$$
\begin{aligned}
C= & \operatorname{erfc}\left(\frac{\eta}{2}\right)+\frac{\eta}{2 \sqrt{\pi}} \int_{1}^{\Delta} \frac{1}{\sigma^{3 / 2}} \exp \left(-\frac{\eta^{2}}{4 \sigma}\right) \\
& \times \operatorname{erfc}\left[\frac{1}{2} \sqrt{\frac{\Delta-1}{\Delta-\sigma}}\left(\xi+\frac{\sigma-1}{\beta}\right)\right] \mathrm{d} \sigma .
\end{aligned}
$$

Several dimensionless parameters are defined in the above equation as: $\eta=y / \sqrt{D_{v}}, \Delta=D_{\mathrm{gb}} / D_{v}$, $\beta=(\Delta-1) \delta /\left(2 \sqrt{D_{v} t}\right)$, and $\xi=(x-\delta / 2) / \sqrt{D_{v} t}$.

Eq. (10) was evaluated with all parameters the same as those chosen in our simulations. For example, the thickness of the grain boundary $\delta$ should be determined from the diffuse-interface field variable profile [7]. Fig. 2(c) shows one example of the average concentration $\langle C\rangle$ as a function of $y^{6 / 5}$ at 3 different time steps. We observed a good agreement between the simulation results and the analytical Whipple's solution, especially at long times in a type- $B$ kinetics where the Whipple's solution is valid [17]. However, at short times $(t=2000)$ there is a little difference between the simulated $\langle C\rangle$ and the calculated $\langle C\rangle$ because the condition $\delta \ll\left(D_{v} t\right)^{1 / 2}$ is not well satisfied at short times. The plot of the logarithm of the average concentration versus $y^{6 / 5}$ was the basis for experimental measurements of grain boundary diffusion coefficients [12].

Based on the simple examples discussed above, the numerical model works quite well in all three regimes of grain boundary diffusion. Although for the idealized Fisher's model, analytical solutions are possible, it is usually not the case for polycrystals, particularly if one is interested in the spatial distribution of diffusing species in the microstructure. The numerical model proposed in this work can be applied to diffusion through a polycrystal without any further complication. Fig. 3 shows an example of the temporal evolution of concentration during diffusion through a polycrystalline grain structure. The grain microstructure, which was generated from a phase field simulation of grain growth [5], was mapped to the $x-y$ plane. Heterogeneous diffusion effect is clear that the concentration values in the grain boundaries are much higher than those in the grain bulk. As can be seen from the concentration distribution in Fig. 3(a) $(t=50,000)$ and Fig. 3(b) $(t=500,000)$, more species diffused into the microstructure at longer diffusion times.

The diffusion coefficient in the grain microstructure is written as $D=D^{*}\left(1-a \sum_{i} \eta_{i}^{2}\right)$ where $D^{*}$ and $a$ are positive constants. Different ratio of grain boundary diffusion coefficient $D_{\mathrm{gb}}$ and bulk diffusion coefficient $D_{v}$ can be easily obtained by 


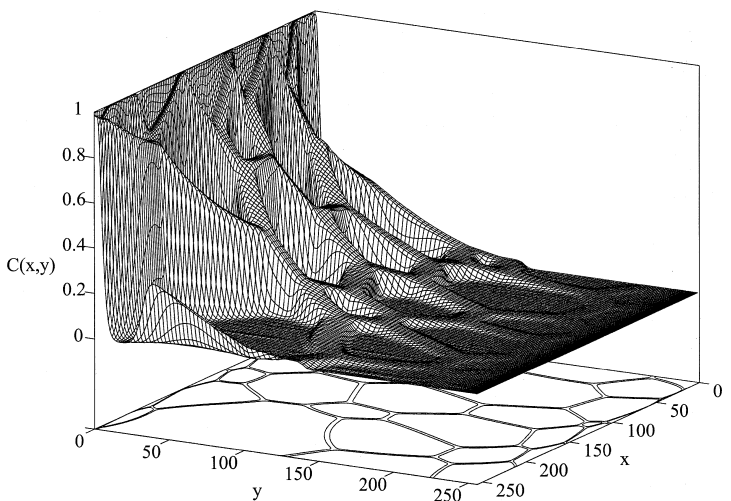

(a)

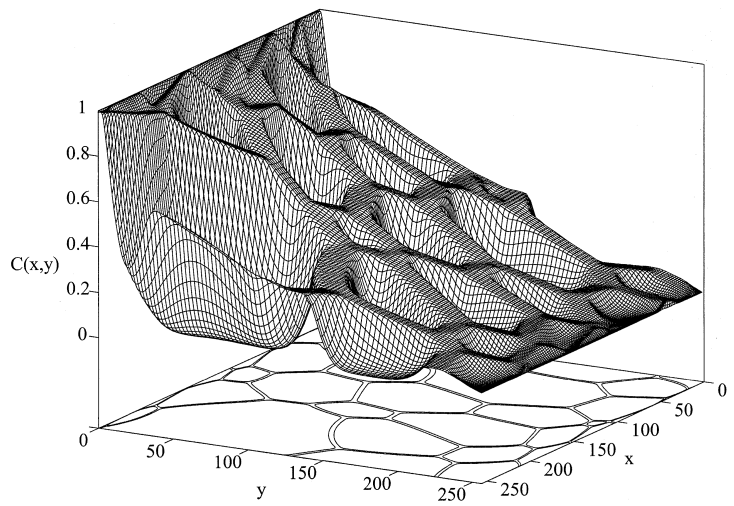

(b)

Fig. 3. Temporal evolution of concentration through a polycrystalline microstructure at two different times, $D=$ $D^{*}\left[1-a \sum_{i} \eta_{i}^{2}\right], D^{*}=1$ and $a=0.9999$ : (a) $t=50,000$; (b) $t=$ 500,000 .

adjusting the value of $a$. Fig. 4 shows the concentration profile through a polycrystalline material for two ratios of $D_{\mathrm{gb}} / D_{v}$ at the same diffusion time. The ratio in Fig. $4(\mathrm{~b})\left(D_{\mathrm{gb}} / D_{v} \approx 20\right)$ is higher than in Fig. 4(a) $\left(D_{\mathrm{gb}} / D_{v} \approx 2.5\right)$, and thus the concentration of the diffusing atoms in the grain boundary differs more considerably from that in the grain bulk.

\subsection{Effective diffusion coefficients of microstruc- tures}

The effective properties of a microstructure, such as electrical conductivity, dielectric constant, magnetic permeability, elastic moduli, thermal

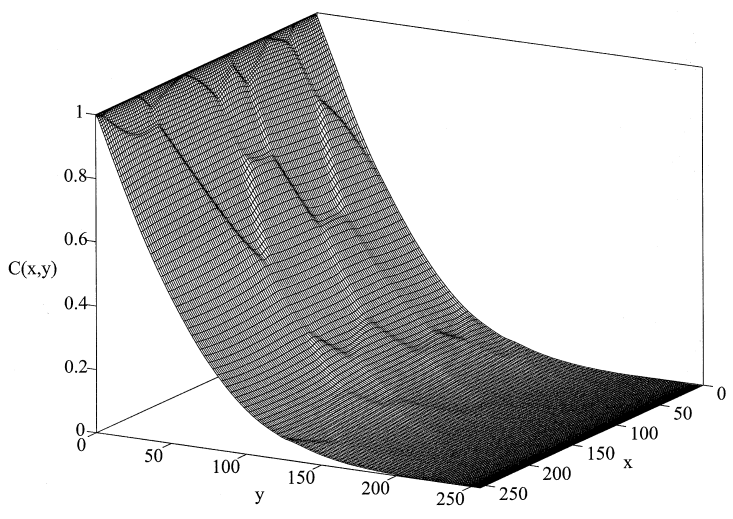

(a)

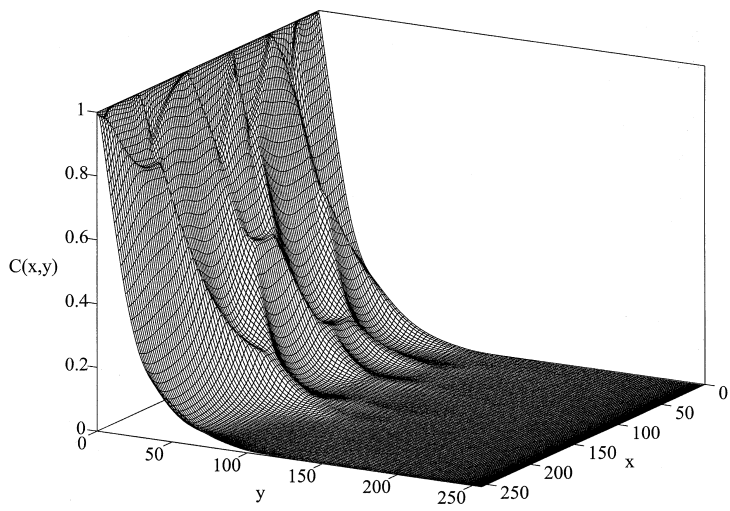

(b)

Fig. 4. Concentration profile in a polycrystal for two ratios of $D_{\mathrm{gb}} / D_{v}$ at the same diffusion time $t=10,000, D=D^{*}(1-$ $a \sum_{i} \eta_{i}^{2}$ ), $D^{*}=1$ : (a) $a=0.8$; (b) $a=0.98$.

conductivity and diffusion coefficient, were often approximated using the so-called mixture rules $[18,19]$

$K_{\text {eff }}^{n}=\sum_{i} V_{i} K_{i}^{n}, \quad-1 \leqslant n \leqslant 1$,

where $K_{\text {eff }}$ is the effective property of the microstructure, $K_{i}$ and $V_{i}$ are the corresponding property and volume fraction of the $i$ th phase. More rigorous theories can predict the upper and lower bounds for the properties $[2,18]$. Recently, there has been increasing interest in directly calculating effective properties of digitized experimental microstructures or microstructures generated from 
computer models [3]. Most of these numerical approaches aimed at approximate solutions of the steady-state equations using finite element or finite different methods [3].

It is easy to show that one can derive the effective properties of a microstructure from the temporal concentration profiles by solving the non-steady-state diffusion equation. We employed two approaches to extract the effective diffusion coefficient $D_{\text {eff }}$ for any given microstructure. One is studying the average concentration profile $\langle C\rangle$ as a function of diffusion distance $y$ at short diffusion times. Assuming Eq. (8) describes the relationship between $\langle C\rangle$ and $y$, we can approximately calculate $D_{\text {eff }}$ by fitting diffusion data to Eq. (8). Another approach is to derive $D_{\text {eff }}$ from the steady-state solution of the non-steady-state diffusion equation at longer intervals of time. The flux $J$ for long diffusion time is related to the concentration gradient by Fick's first law,

$J=-D_{\text {eff }} \nabla C$.

The total flux $J$ can be obtained easily from the diffusion coefficient distribution $D(\mathbf{r})$ and the concentration distribution $C(\mathbf{r})$ through the microstructure at the steady state. $\nabla C$ in Eq. (12) is determined by the applied concentration gradient. Our numerical work shows $D_{\text {eff }}$ computed from Eqs. (8) and (12) has a difference less than 2\%, which can satisfy most of the practical applications. One of the main advantages of this approach is that it produces both the time-dependent concentration profiles in a microstructure and the effective properties of the microstructure. In addition, it avoids any complicated boundary conditions and thus allows one to study the diffusion transport through any arbitrary microstructures.

As test examples, we first computed $D_{\text {eff }}$ for two of the simplest possible microstructures. The system is composed of parallel slabs of A and B with different diffusion coefficients $D_{\mathrm{A}}$ and $D_{\mathrm{B}}$, shown in Fig. 5. In Fig. 5(a), the flux is parallel to the plane of the slabs, and the arrangement is equivalent to a parallel electrical circuit. Each slab has the same concentration gradient, and most of the flux is through the component with higher diffusivity. $D_{\text {eff }}$ is given by $D_{\text {eff }}=D_{\mathrm{A}} V_{\mathrm{A}}+D_{\mathrm{B}} V_{\mathrm{B}}$, where $V_{\mathrm{A}}$ and $V_{\mathrm{B}}$ are the volume fraction of each component. This corresponds to $n=1$ in Eq. (11). In contrast, for diffusion flux perpendicular to the plane of the slabs in Fig. 5(b), it is equivalent to a series electrical circuits. The flux through each slab is equal, but the concentration gradients are different. In this case, $n=-1$ in Eq. (11) and $D_{\text {eff }}=D_{\mathrm{A}} D_{B} /$ $\left(D_{\mathrm{A}} V_{\mathrm{B}}+D_{\mathrm{B}} V_{\mathrm{A}}\right)$. Fig. 6 shows the comparison of calculated and theoretical $D_{\text {eff }}$ as a function of volume fraction for these two parallel slab microstructures. Slab arrangements in Fig. 5(a) and (b) are indicated by $\|$ and $=$ respectively. As can be seen from the graph, the effective diffusion coefficients computed agree well with those of theoretical solutions for both cases, thus validating our methods. Generally $D_{\text {eff }}$ for the two parallel slab microstructures will cover the complete range of

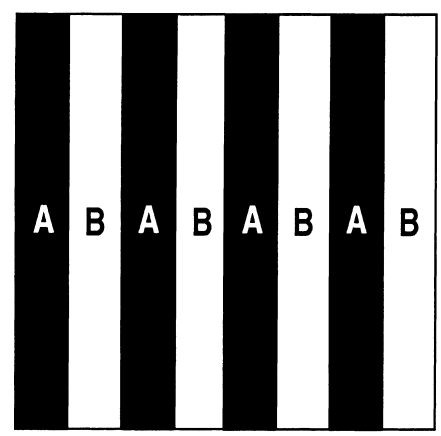

(a)

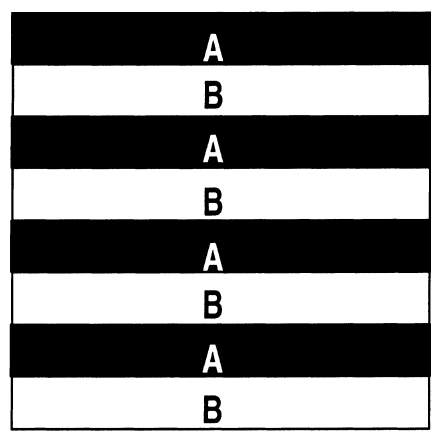

(b)

Fig. 5. Idealized two-phase microstructures - parallel slabs: (a) flux parallel to the plane of slabs (\|); (b) flux perpendicular to the plane of slabs $(=)$. 


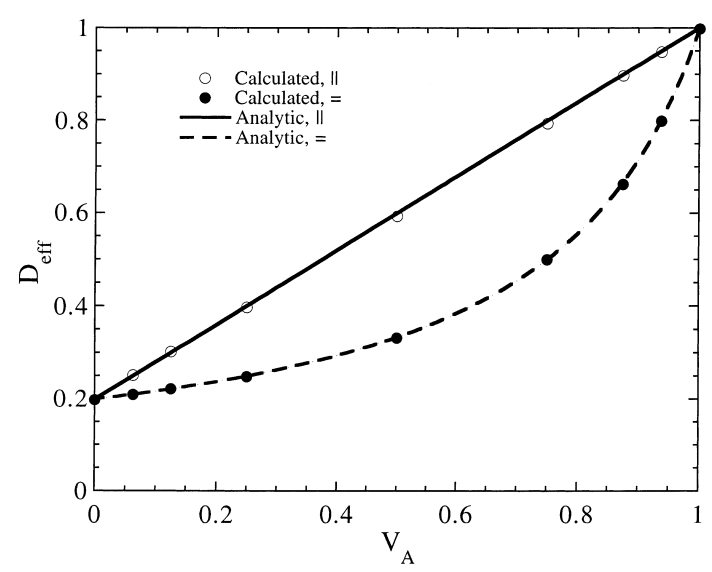

Fig. 6. Computed and theoretical effective diffusion coefficient $D_{\text {eff }}$ as a function of volume fraction of phase A for parallel slab microstructures, $D_{\mathrm{A}}=1.0, D_{\mathrm{B}}=0.2$.

effective properties between the upper and lower bounds as shown in the solid line and dashed line in Fig. 6. It would be expected that for arbitrary microstructures, $D_{\text {eff }}$ was within this range.

For a polycrystalline material, diffusion was carried out as described in the previous section. In particular, we examined the effect of the average grain size $d$ of a grain structure on the effective diffusion coefficient. The results are plotted as the filled circles shown in Fig. 7, where $D_{\text {eff, simulation }}$ is calculated from steady state concentration profile. $D_{\text {eff,simulation }}$ varies inversely with the average grain

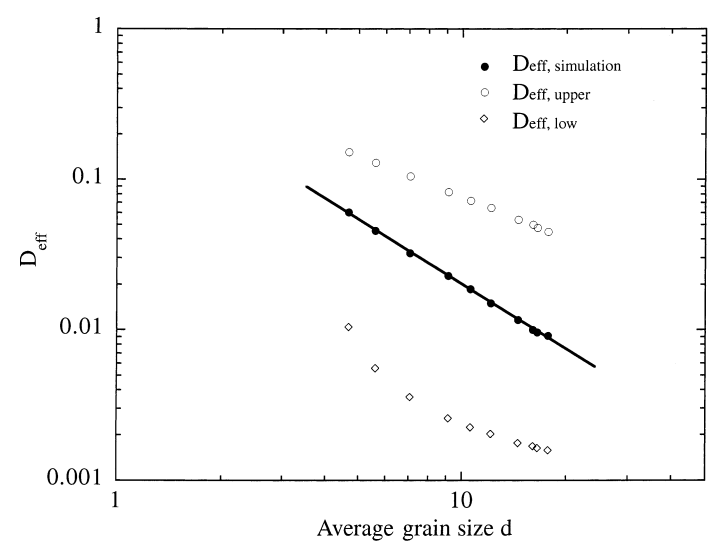

Fig. 7. Effective diffusion coefficient $D_{\text {eff }}$ as a function of average grain size $d$ for a single-phase polycrystalline microstructure, $D=1-0.999 \sum_{i} \eta^{2}$. $D_{\text {eff, upper }}$ and $D_{\text {eff, low }}$ are the effective diffusion coefficients predicted by mixture rules. size $d$. This result is in good agreement with that of sharp-interface simulations by Swiler et al. [14]. The driving force for grain growth is the reduction of grain boundary energy. As the grain size becomes larger, the volume fraction of grain boundaries decreases, resulting in a decrease in the effective diffusion coefficient. In Fig. 7, we also plotted the theoretical effective diffusion coefficients $D_{\text {eff,upper }}$ and $D_{\text {eff,low }}$ as the upper and low bounds of the effective diffusion coefficients which can be predicted by rule of mixing as $D_{\mathrm{gb}} f_{\mathrm{gb}}+$ $D_{v}\left(1-f_{\mathrm{gb}}\right)$ and $D_{\mathrm{gb}} D_{v} /\left[D_{\mathrm{gb}}\left(1-f_{\mathrm{gb}}\right)+D_{v} f_{\mathrm{gb}}\right]$, respectively, where $f_{\mathrm{gb}}$ is the volume fraction of grain boundaries. However, for a diffuse-interface description of the microstructure, there is some ambiguity in determining the exact values of $D_{\mathrm{gb}}, D_{v}$ and $f_{\mathrm{gb}}$. Recognizing that $D_{\text {eff }}$ predicted by rule of mixing is essentially a volume average, we calculated $D_{\text {eff,upper }}$ as $(1 / N) \sum_{\mathbf{r}} D(\mathbf{r})$ where $N$ is the total number of lattice points. Similarly $D_{\text {eff, low }}$ was computed as $N /\left(\sum 1 / D(\mathbf{r})\right)$. As expected, for all the grain sizes studied, $D_{\text {eff,simulation }}$ is between $D_{\text {eff, upper }}$ and $D_{\text {eff,low }}$, which are the effective diffusion coefficients for idealized grain structures where grain boundaries can be treated as parallel slabs embedded in the grains. The difference between $D_{\text {eff,simulation }}$ and $D_{\text {eff,upper }}$ was explained by Swiler et al. [15] as a result of triple junctions between grains as bottlenecks to diffusion. Their sharp-interface simulations showed that $D_{\text {eff, simulation }} \approx 0.27 D_{\mathrm{gb}} \delta / d$ [14], where $\delta$ is the grain boundary width and $\delta / d$ is the approximated volume fraction of grain boundaries.

As a final example, diffusion through two-phase microstructures was studied. The two-phase microstructures in Fig. 8 were obtained from our phase field simulations of phase separation of an initially homogeneous system. The two-phase system forms an inter-connected or a dispersed microstructure at different volume fractions. Fig. 9 shows the change of $D_{\text {eff }}$ (obtained from the steady state concentration profile) as a function of volume fraction for these microstructures (plotted with circle symbols). As the volume fraction of phase A with high diffusion coefficient $\left(D_{\mathrm{A}} \gg D_{\mathrm{B}}\right)$ increases, the shape of $A$ droplets changes, and $D_{\text {eff }}$ increases gradually. After $V_{\mathrm{A}}$ reaching about $50 \%$, there is a sharp increase in $D_{\text {eff }}$ when the 


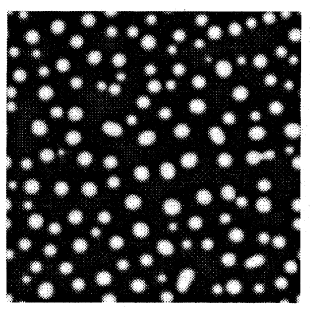

(a)

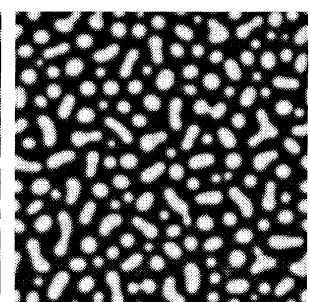

(b)

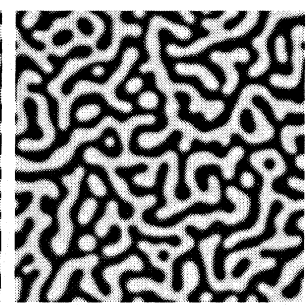

(c)

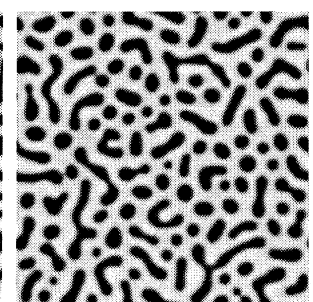

(d)

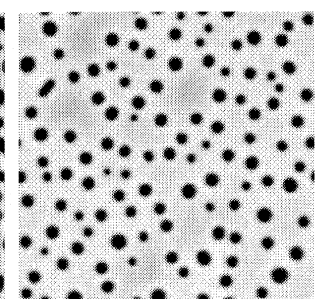

(e)

Fig. 8. Two-phase microstructures for different volume fraction of phase $A$ (shown in white color) showing the percolation transition, (a)-(e) for $V_{A}=16 \%, 32 \%, 50 \%, 62 \%$, and $87 \%$, respectively.

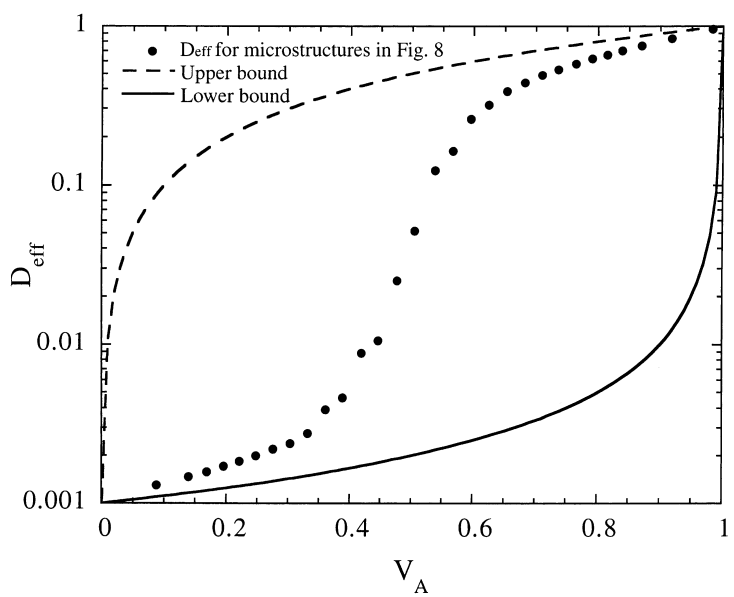

Fig. 9. Effective diffusion coefficient $D_{\text {eff }}$ as a function of volume fraction. Circles were calculated from the microstructures in Fig. 8. Dashed line and solid line were plotted from Eq. (11) representing the upper and lower bounds of $D_{\text {eff }}$. $D_{\mathrm{A}} / D_{\mathrm{B}}=1000$.

microstructure changes from isolated phase A in B to inter-connected $\mathrm{A}$ and $\mathrm{B}$ phases. The ratio of $D_{\mathrm{A}} / D_{\mathrm{B}}$ is three orders of magnitude in Fig. 9. The dashed line and solid line represent the upper and low bounds of $D_{\text {eff }}$ determined from Eq. (11) as $n=1$ and -1 , respectively. It is usually observed that the larger the distinction between the properties of different phases in heterogeneous systems, the more obvious is the percolation transition [18].

Our numerical experiments have demonstrated that the Fourier-Chebyshev spectral method is very efficient for solving the time-dependent diffusion equation in which the diffusivity distribution is a smooth function throughout the microstructure, especially for microstructures described by the diffuse-interface model. The excellent agreement between our diffuse-interface simulation and sharp-interface analytical or numerical methods showed that diffuse-interface model is very effective for describing diffusional transport in mesoscale microstructures. In recent years, the diffuse-interface phase-field approach has been recognized as a powerful mathematical model for simulating the mesoscale morphological pattern formation such as grain growth and Ostwald ripening. By coupling the phase-field modeling of microstructure evolution, the approach developed in this paper allows us to model the effective diffusivity evolution in an evolving microstructure. More details will be reported in the near future.

\section{Conclusion}

We have simulated the temporal mass diffusion transport through an arbitrary microstructure based on a phase-field approach. Diffusion coefficients were calculated as a function of the order parameters describing a single-phase grain structure where grain boundary diffusion was much higher than bulk diffusion and a two-phase microstructure where the diffusivities varied with the phase. The semi-implicit Fourier-Chebyshev spectral method was shown to be accurate and efficient in solving the variable diffusion equations. The accuracy of the model was demonstrated by reproducing the classic diffusion results of grain boundary diffusion in all three kinetic regimes 
(type A, B and C) as well as the parallel slabs geometries. We demonstrated that one can extract effective diffusion coefficient of a microstructure from the temporal diffusion profiles. Furthermore, the sharp interface results of Swiler et al. were reproduced to show that the effective diffusivity varies inversely with grain size. This model was used to predict the effective diffusivity of twophase percolating and non-percolating structures where each phase has very different diffusivities. This approach can be also used to study similar transport problems such as heat transport by recognizing the similarity between mass diffusion and thermal diffusion equations.

\section{Acknowledgements}

The authors are grateful for the financial support from the Sandia National Laboratory and the National Science Foundation under Grant No. DMR 96-33719 and DMS 9721413.

\section{References}

[1] I. Kaur, Y. Mishin, W. Gust, Fundamentals of Grain and Interphase Boundary Diffusion, third ed., Wiley, Chichester, 1995.
[2] S. Torquato, Int. J. Solids Struct. 35 (1998) 2385.

[3] E.J. Garboczi, D.P. Bentz, Constr. Building Mater. 10 (1996) 293.

[4] L.Q. Chen, Y.Z. Wang, JOM 48 (1996) 13.

[5] D.N. Fan, L.Q. Chen, S.P. Chen, Mater. Sci. Eng. A 238 (1997) 78

[6] C. Canuto, M.Y. Hussaini, A. Quarteroni, T.A. Zang, Spectral Methods in Fluid Dynamics, Springer, Berlin, 1987.

[7] L.Q. Chen, J. Shen, Comput. Phys. Commun. 108 (1998) 147.

[8] J. Shen, SIAM J. Sci. Comput. 16 (1995) 74.

[9] J.C. Fisher, J. Appl. Phys. 22 (1951) 74.

[10] R.T.P. Whipple, Philos. Mag. 45 (1954) 1225.

[11] T. Suzuoka, J. Phys. Soc. Jpn. 19 (1964) 839.

[12] H.S. Levine, C.J. MacCallum, J. Appl. Phys. 31 (1960) 595.

[13] X. Gui, S.K. Dew, M.J. Brett, J. Appl. Phys. 74 (1993) 7173 .

[14] T.P. Swiler, V. Tikare, E.A. Holm, Mater. Sci. Eng. A 238 (1997) 85.

[15] T.P. Swiler, E.A. Holm, Ceram. Trans. 69 (1997) 203.

[16] L.G. Harrison, Trans. Faraday Soc. 57 (1961) 1191.

[17] J. Philebert, Atom Movements - Diffusion and Mass Transport in Solids, Les Editions de Physique, Les Ulis, 1991.

[18] C.W. Nan, Prog. Mater. Sci. 37 (1993) 1.

[19] L.E. Nielsen, Predicting the Properties of Mixtures: Mixture Rules in Science and Engineering, Marcel Dekker, New York, 1978. 